\title{
Wideband Channel Measurements and First Findings for Low Altitude Drone-to-Drone Links in an Urban Scenario
}

\author{
Dennis Becker, Uwe-Carsten Fiebig and Lukas Schalk \\ Institute of Communications and Navigation \\ German Aerospace Center (DLR) \\ Oberpfaffenhofen-Wessling, Germany \\ dennis.becker@dlr.de
}

\begin{abstract}
In order to prevent collisions between unmanned aerial vehicles (UAVs), Drone-to-Drone (D2D) communication is a promising technology for enabling reliable collision avoidance systems. For a high reliable communication system accurate channel models, specifically designed for UAVs in typical environments, are needed. Especially, the urban environment for small sized UAVs in very low level (VLL) airspace is challenging due to rich multipath propagation and non line-ofsight (NLOS) conditions. In order to model the real world propagation behavior, channel measurements with small UAVs in different urban scenarios are mandatory. So far, no wideband channel model based on measurements for D2D communication has been proposed. Therefore we conducted channel measurements with our MEDAV RUSK-DLR channel sounder with a $100 \mathrm{MHz}$ bandwidth sounding signal at $5.2 \mathrm{GHz}$ and two flying hexacopters. A key feature in our D2D wideband channel measurement campaign was the use of optical fibers. In this paper, we present the scenarios of our campaign and show first findings. By applying a geometrical signal path simulation we identify multipath component (MPC) sources. The measurements reveal that the D2D MPC scenario is clearly three-dimensional.

Index Terms-Unmanned Aerial Vehicle, Air-to-Air, Propagation, Drone-to-Drone Communication, Channel Measurement, Simulation
\end{abstract}

\section{INTRODUCTION}

In the near future, the number of UAVs in VLL airspace is expected to increase significantly due to manifold applications like inspection of critical infrastructure, parcel delivery or air taxis. In the longterm we foresee a UAV-to-person ratio of around one, resulting in densities of hundreds of airborne UAVs per square kilometer in cities during rush hours. For a safe integration of UAVs into urban airspace, high reliable collision avoidance systems are mandatory. We are convinced that D2D communication is a promising approach for sharing information on UAV trajectories in a reliable manner and with very low latency. We expect that D2D communication channels for UAVs exhibit rich multipath characteristics in three dimensions and, thus, may be even more challenging than vehicular channels. Channel measurements are inevitable in order to derive accurate channel models for D2D communication links.
A propagation measurement campaign with small ${ }^{1}$ sized UAVs is a big challenge since we have strong limitations on the payload in terms of size, weight and power consumption. Thus sophisticated wideband D2D channel measurements are difficult to achieve as long as transmitter and receiver equipment is carried by UAVs. Consequently, only largescale parameters have been investigated so far for the D2D channel with small sized UAVs with IEEE802.11 radios [1] and IEEE802.15.4 radios [2]-[4].

Another challenge is to fly UAVs in urban scenarios in different heights and close to buildings. These scenarios are of great interest, but to our knowledge they have not yet been the subject of a propagation measurement campaign. Therefore, other methods have been applied so far to gain a first understanding about the D2D channel: [5] describes the propagation characteristics of the air-to-air (A2A) channel in an urban environment with a ray-tracing simulation considering largescale parameters.

To overcome the payload limitations of small UAVs, we proposed a measurement setup that extends the operation of channel sounding equipment by exploiting analog optical links to send the channel sounding signal to the drone. [6]. With our MEDAV RUSK-DLR channel sounding equipment and the proposed extension we conducted D2D channel measurements at $5.2 \mathrm{GHz}$ with $100 \mathrm{MHz}$ bandwidth for urban scenarios. The used frequency band has been chosen since the future control and non payload communication (CNPC) link is planned to operate in the C-band $(5.03 \mathrm{GHz}$ to $5.091 \mathrm{GHz})$ [8].

The objective of this paper is to give an overview about all scenarios of our D2D channel measurement campaign. Furthermore we will present first findings of one of the assessed scenarios where two hexacopters fly between buildings at different heights. In order to understand where the many multipath signals originate from, we also derive the geometrical signal paths of all major MPCs based on the exact trajectories of the involved UAVs and the three-dimensional

\footnotetext{
${ }^{1}$ The classification small is typically used for UAVs, which weigh less than
} 25 kilogram [7] 


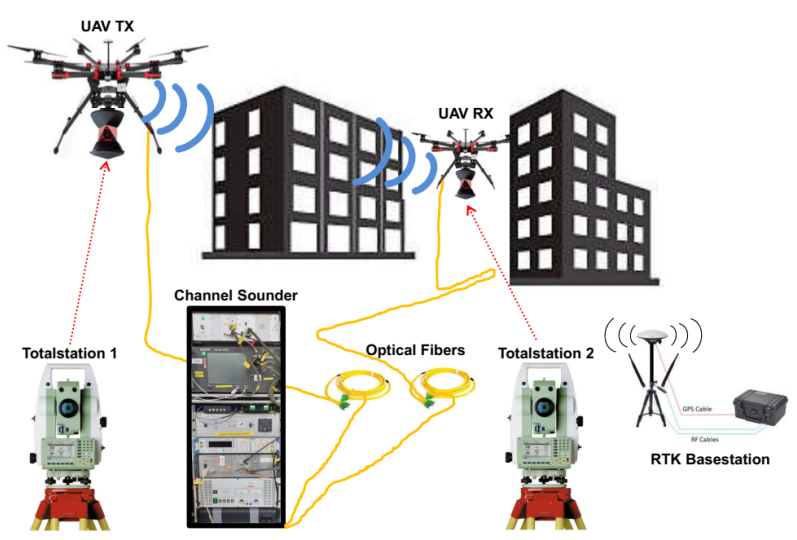

Fig. 1. Overview of the measurement setup.

layout of the scenario.

\section{Measurement CAmpaign Description}

With our proposed channel sounding setup in [6] we conduct D2D channel measurements with different scenarios at our site in Oberpfaffenhofen Germany, which provides an urban scenario with tall buildings standing close by. For the campaign we identified different environments and scenarios described further in II-A. The overall goal of this campaign is to characterize the specific large-scale and small-scale characteristics for the D2D communication channel between small UAVs in an urban environment and to identify and locate possible MPCs in the three-dimensional layout.

We have already carried out several test flights with two hexacopters and recorded preliminary measurement data in order to evaluate our hardware setup and the flight behavior of our UAVs. In this work we use these measurements to investigate first MPC sources.

\section{A. Planned Scenarios}

We conduct measurements at three different environments at our site that allow for different scenarios with line of sight (LOS) and NLOS conditions at different heights, speeds and trajectories. Figure 2 illustrates the environments A, B and $\mathrm{C}$ with the planned flight paths for the drones. We are considering following scenarios.

A1 - Horizontal Flight with LOS Condition: In this scenario at environment A, one drone flies horizontally on line path AP1 at constant height, while the other drone hovers at fixed position at point path AP2. Thereby the drones are always in LOS. With this scenario, we tested our hardware setup and evaluated the flight behavior of the drone when flying at relatively low height between a narrow urban canyon with a width of approximately $7 \mathrm{~m}$, which is the most challenging flight mission in our our campaign.

A2 - Horizontal Flight with NLOS Condition: In this scenario at environment A, one drone flies horizontally on line path AP1 at constant height, while the other drone flies horizontally on line path AP3. Thereby the drones are always in NLOS condition as the view is obstructed by the building in between.

A3 - Collision Course at Corner: For Scenario A3 one drone flies path AP1, while the other drone flies at path AP4. Thereby the drones fly towards and away from each other with changing LOS conditions.

A4 - Balcony: For Scenario A4 one drone is placed on a balcony of building B103 at point AP6, while the other drone flies at path AP5 over the building and along path AP4.

B1 - Horizontal Flight with LOS Condition: In this scenario at environment $\mathrm{B}$, one drone flies horizontally at path $\mathrm{BP} 3$, while the other drone hovers at point BP2. The building close by offers an interesting surface due to big window shutters for sun protection.

B2 - Vertical Flight in Courtyard: In this scenario at environment B, one drone flies horizontally at path BP3, while the other drone flies vertically at point BP1 down and up in an inner courtyard.

C1 - Horizontal Fast Flight with Changing Environment: For Scenario $\mathrm{C} 1$ one drone flies path CP1 horizontally, while the other drone hovers at points $\mathrm{CP} 2$ and $\mathrm{CP} 3$. This environment allows for higher velocities of the drones due to much more open space.

\section{B. Measurement Setup}

Our measurement setup consists of seven basic components shown in figure 1 . We are using two custom-build DJI S900 hexacopters that are connected to our MEDAV RUSKDLR channel sounder [9] via $600 \mathrm{~m}$ long optical fibers. By guiding the sounding signal trough optical fibers we extend the operation of our channel sounder and enable wideband channel measurements between flying drones. In [6] we proposed a channel sounding setup and explain our equipment in more detail. Table I summarizes important parameters. We are transmitting an OFDM-like multitone signal at $5.2 \mathrm{GHz}$ with $100 \mathrm{MHz}$ bandwidth and $30 \mathrm{dBm}$ power over omnidirectional linear polarized dipole antennas. The receiving and transmitting antennas are vertically aligned and placed at the bottom of the drones. In order to precisely navigate the drones, a RTK system, consisting of a static basestation on the ground and receivers on the drones, was used. Our flight trials revealed that a single GNSS solution on the drones is insufficient for autonoumusly flying waypoint missions safely at lower heights between the buildings. For an accurate position tracking of the drones we use two Leica TCRP1201 total stations that allow us to track them with position uncertainties of a few centimeters depending on the speed of the drones. The Leica stations are tracking prisms attached on the bottom of the drones, where they can be seen from the Leica stations at ground during the 


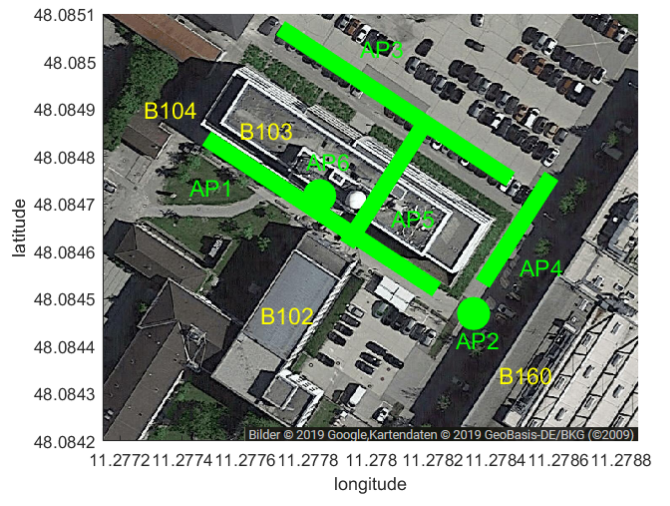

(a) Environment A

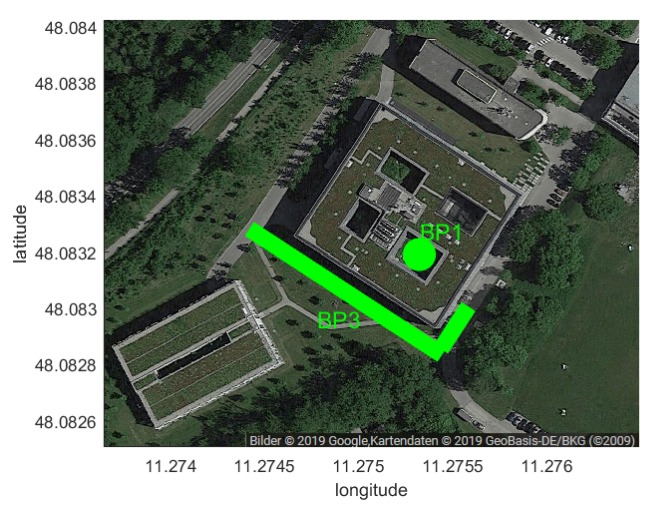

(b) Environment B

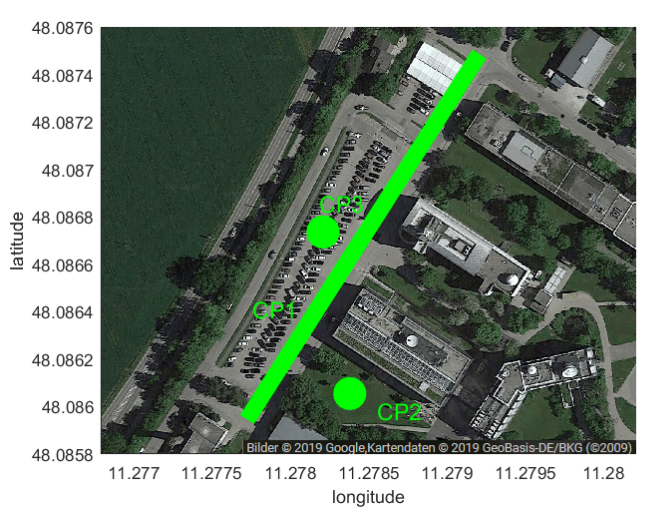

(c) Environment $\mathbf{C}$

Fig. 2. Three environments for several measurement scenarios with planned flight paths for the drones.

flight. The Leica stations must be placed carefully in order to ensure LOS condition for the whole measurement and to keep the angular velocity as small as possible, as it is restricted due to mechanical limits.

\section{First Measurements}

For first experiences with our setup we started with measurements for the described scenario A1 at two different heights. Figure 3 illustrates the planned waypoint missions and
TABLE I

Channel Sounding Parameter

\begin{tabular}{lcl}
\hline Parameter & Symbol & Value \\
\hline \hline Center frequency & $f_{c}$ & $5.2 \mathrm{GHz}$ \\
Bandwidth & $B$ & $100 \mathrm{MHz}$ \\
Tx Power & $P_{\mathrm{tx}}$ & $30 \mathrm{dBm}$ \\
Signal duration & $T_{p}$ & $12.8 \mu \mathrm{s}$ \\
Signal period & $T_{g}$ & $1.024 \mathrm{~ms}$ \\
Max resolvable doppler frequency & $f_{d_{\max }}$ & $488 \mathrm{~Hz}$ \\
ADC resolution & $r e s_{\mathrm{adc}}$ & $8 \mathrm{bit}$ \\
Dynamic range & $\alpha_{\mathrm{agc}}$ & $52 \mathrm{~dB}$ \\
Antenna Tx & & omni-dir. V-pol. 0 dBi \\
Antenna Rx & & omni-dir. V-pol. 0 dBi \\
\hline
\end{tabular}

the tracked positions for the drones as well as for the Leica station positions. In the first measurement the drones flew both at about $30 \mathrm{~m}$ height above ground level, which is higher than the surrounding buildings. In the second measurement they flew at about $15 \mathrm{~m}$ height, which is below the rooftops.

For the two scenarios (A1 at height $15 \mathrm{~m}$ and $30 \mathrm{~m}$ ), figures $5 \mathrm{a}$ and $6 \mathrm{a}$ show the channel impulse responses for both flights. In both figures the strong LOS signal can be clearly seen. It's delay increases and decreases constantly as the moving drone flies towards and away from the hovering drone. Furthermore strong reflections from MPCs with long and short life times can be seen. In the following we investigate these MPCs and describe possible sources.

\section{Geometrical Signal Path Simulation}

In order to understand the channel impulse characteristics of two measurements explained in II-C, we simulate geometrical signal paths based on the exact trajectories of the UAVs and the three-dimensional layout of the scenario A1 with all buildings close by (B102, B103, B104, B160). Figure 4 illustrates the modeled environment with the tracked trajectories of both

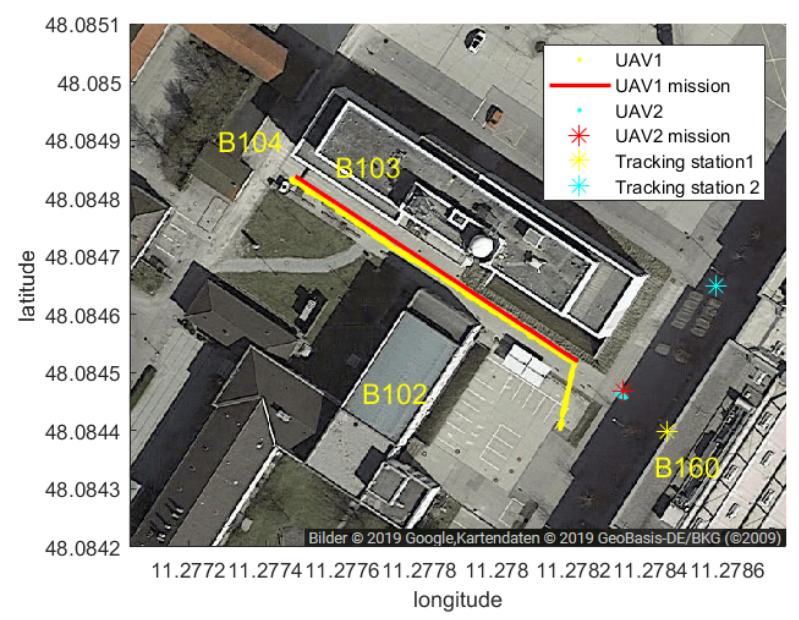

Fig. 3. Flight trial for D2D channel measurement. In this scenario UAV1 flies a line mission $65 \mathrm{~m}$ in an urban canyon two times back at $15 \mathrm{~m}$ height. UAV2 hovers at same height and is always in LOS. 


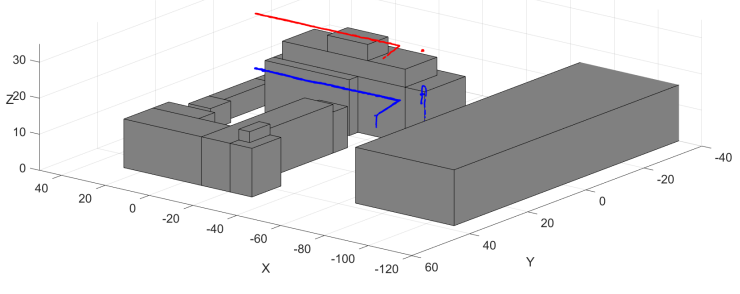

Fig. 4. Modeled simulation environment with buildings close by in scenario A1 and tracked drone positions when flying at $15 \mathrm{~m}$ and $30 \mathrm{~m}$ height.

UAVs and both flights (blue: $15 \mathrm{~m}$ and red: $30 \mathrm{~m}$ height) in the local Cartesian coordinate system.

For every measured position of the UAVs we estimate the delays and the received signal power relative to the LOS signal for several signal paths with single and double reflections on the building and ground surfaces. Then we compare the results with the measurements. For the delays we assume speed of light and calculate the geometrical distances of the reflected signal path on the surfaces with the well known two-ray ground reflection model and apply it for the vertical surfaces of the buildings as well. The double bounce propagation distances are calculated by mirroring the signal source points at one of the two involved surfaces as virtual signal sources. For the received signal powers we only assume free space path loss and do not consider losses by different reflection coefficients as the results shall only help to identify the MPCs. For better comparison with the measurements, we quantize the results with the same signal period time and delay resolution.

In addition we show possible sources for the MPCs not assumed a priori by calculating the MPC regions with same signal delays and intersecting them with satellite maps. For this we simply assume all MPCs located horizontally on an ellipse around the transmitter and receiver as foci points like done in [10].

\section{RESULTS AND COMPARISON}

Fig. $5 \mathrm{~b}$ and $6 \mathrm{~b}$ show the results of the estimated channel impulse response over time by simulation. The simulated long life-time reflections can be found in the measurements, but do not explain all the reflections that are visible. They are named after the sources of the reflections, which are the surrounding buildings (B102, B103, B104 and B160) as well as the ground surface (GREF). There are single bounce (SREF) and double bounce (DREF) propagations on the surfaces. The strong LOS component can be seen very clearly with a linear increasing and decreasing delay as the receiving drone moves down and up the corridor at constant speed.

In both measurements there are two stronger reflections that were not estimated by simulation. They are clearly visible in fig. $6 \mathrm{~b}$ at around $460 \mathrm{~ns}$ and $510 \mathrm{~ns}$ around minute 3 , when the flying drone is at it's furthest distance to the hovering drone. In order to estimate possible locations for the origins

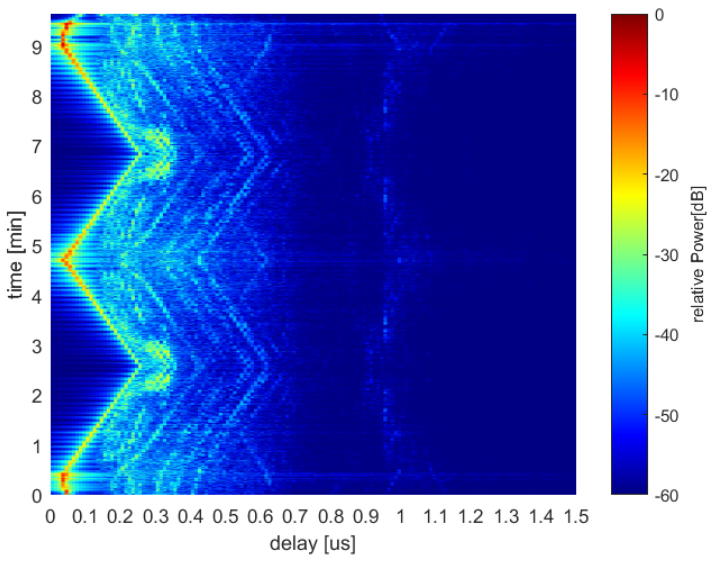

(a) Measured channel impulse response over time with received power relative to LOS signal power .

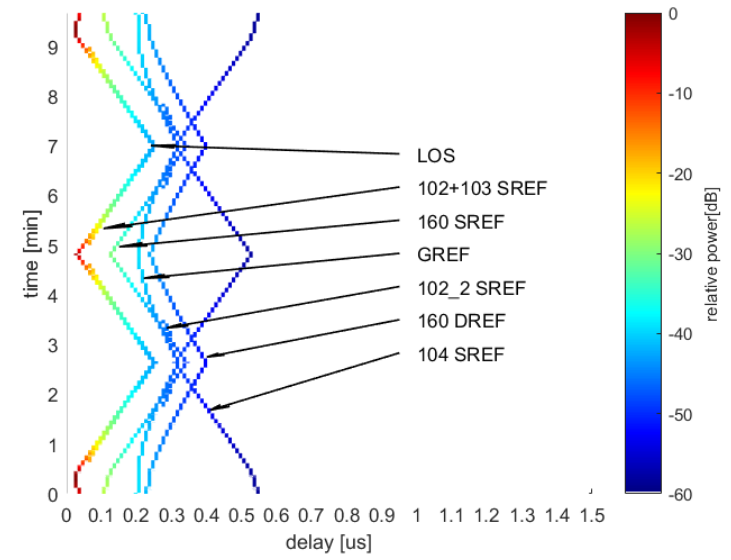

(b) Estimated channel impulse response over time by simulation .

Fig. 5. Flight scenario A1 at $30 \mathrm{~m}$ height.

of these reflections, we plotted the regions in yellow of same signal delays around the receiver in fig. 7 on a satellite-map. For the indicated yellow regions we then identified the red regions as possible MPC sources of the reflections as there are steel beams on the rooftop of building 160 that intersect with the yellow regions. Also the steady increasing delay in same manner as the LOS delay shows, that the MPCs must be located somewhere in the opposite of the flight direction.

When comparing the two different measurements, it can be seen that all reflections at the grounds surface show different delays as the height of the drones is different in both cases. Delays of the single bounce propagations on the building surfaces stay nearly the same. Furthermore the flight scenario A1 at height $30 \mathrm{~m}$ shows a reflection from a higher part of the roof of building B102 (102_2 SREF) that is not present at height $15 \mathrm{~m}$. This is simply because this surface is not in LOS for the signal receiving drone at lower heights. The reflections resulting from building B104 are visible if not overlaid by stronger reflections. 


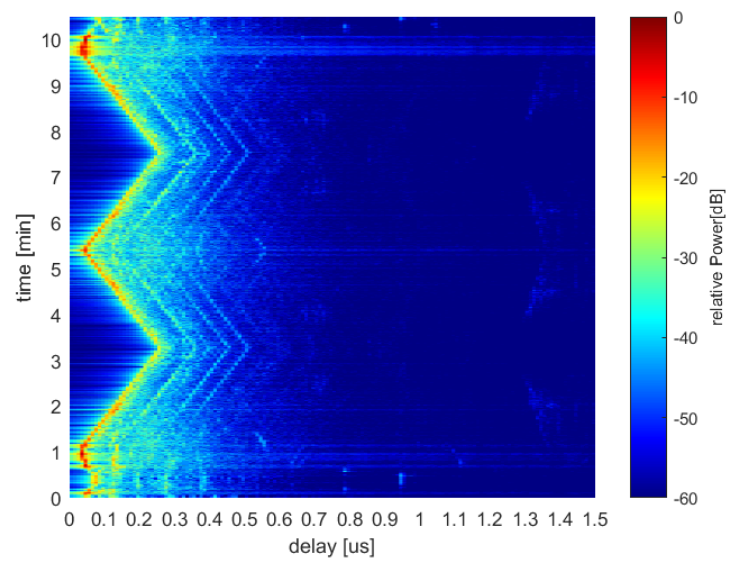

(a) Measured channel impulse response over time with received power relative to LOS signal power.

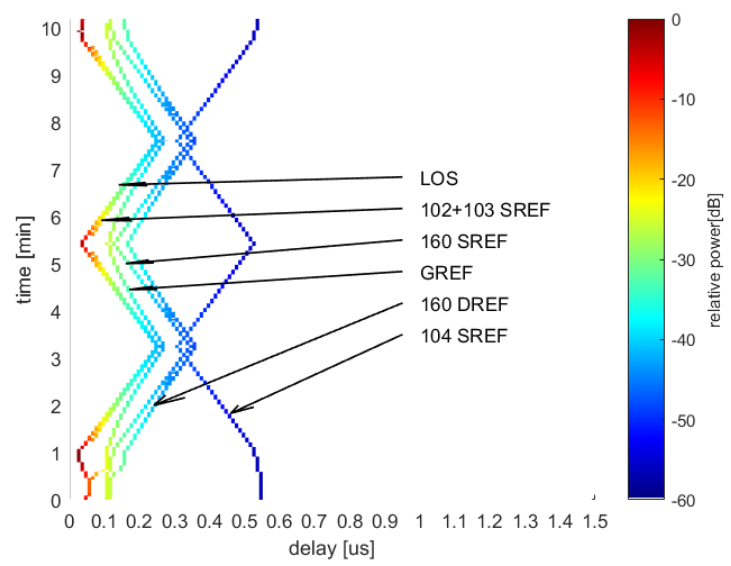

(b) Estimated channel impulse response over time by simulation .

Fig. 6. Flight scenario A1 at $15 \mathrm{~m}$ height.

\section{CONCLUSION AND OUTLOOK}

In this work we showed first findings of wideband channel measurements for low altitude D2D links in an urban scenario. We identified most of the MPC sources by comparing the measurements with simulations of the three-dimensional layout and recorded flight tracks of the drones. The results reveal a rich multipath environment that is clearly threedimensional. MPCs are identified from reflections on the surrounding buildings and the grounds surface. Even double bounce propagations are visible in the measurements and strong reflections from good reflectors like metallic objects on a rooftop. In the next we will further investigate the D2D communication channel with the different measurement scenarios described for our measurement campaign and extend the MPC identification and localization in the three-dimensional space.

\section{ACKNOWLEDGMENT}

The authors thank all colleagues at Institute of Communications and Navigation, German Aerospace Center, who helped to perform several flight trials.

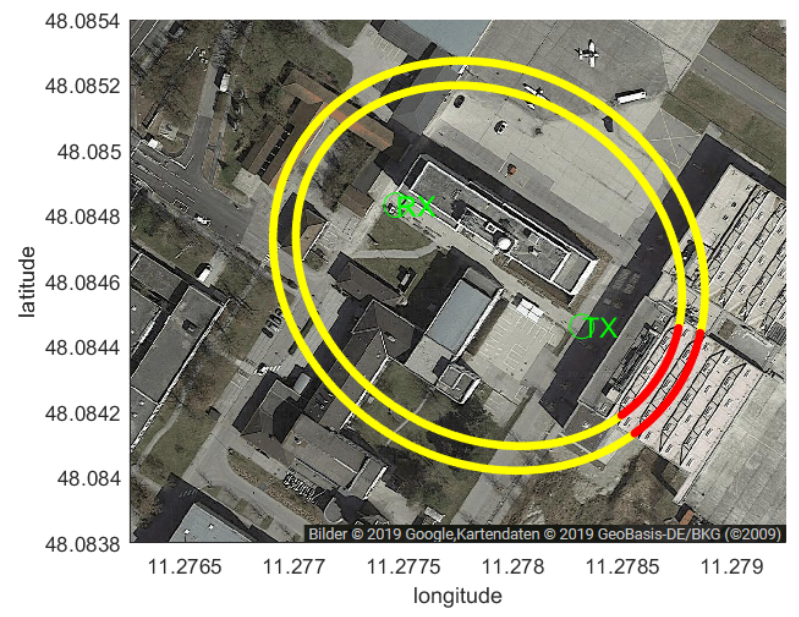

Fig. 7. Possible MPC regions of same signal delays at $510 \mathrm{~ns}$ and $460 \mathrm{~ns}$ around the receiver RX. Red regions illustrate the assumed origins of the signal reflections.

\section{REFERENCES}

[1] N. Goddemeier and C. Wietfeld, Investigation of air-to-air channel characteristics and a UAV specific extension to the rice model, in Proc. IEEE Globecom Workshops, San Diego, CA, USA, Dec 2015, pp. 15.

[2] N. Ahmed, S. S. Kanhere, and S. Jha, On the importance of link characterization for aerial wireless sensor networks, IEEE Communications Magazine, vol. 54, no. 5, pp. 5257, May 2016.

[3] Jude Allred, Ahmad Bilal Hasan, Saroch Panichsakul, William Pisano, Peter Gray, Jyh Huang, Richard Han, Dale Lawrence, and Kamran Mohseni. 2007. SensorFlock: an airborne wireless sensor network of micro-air vehicles. In Proceedings of the 5th international conference on Embedded networked sensor systems (SenSys '07). ACM, New York, NY, USA, 117-129.

[4] Apratim Shaw and Kamran Mohseni, A fluid dynamic based coordination of a wireless sensor network of unmanned aerial vehicles: 3-D simulation and wireless communication characterization,IEEE Sensors J., Special Issue on Cognitive Sensor Networks, 722-736, 2011

[5] Zhou, L., Yang, Z., Zhao, G., Zhou, S., Wang, C-X. (2019). Propagation Characteristics of Air-to-Air Channels in Urban Environments. In 2018 IEEE Global Communications Conference (GLOBECOM) [8647360] (Global Communications Conference (GLOBECOM)).

[6] Becker, Dennis and Schalk, Lukas , Enabling Air-to-Air Wideband Channel Measurements between Small Unmanned Aerial Vehicles with Optical Fibers, AIAA/IEEE 38th Digital Avionics Systems Conference, DASC 2019, 9. -12.September, San Diego, USA.

[7] Federal Aviation Administration, Operation and Certification of Small Unmanned Aircraft Systems, accessed: 2019-07-07. [Online]. Available: https://www.faa.gov/uas/media/RIN_2120-AJ60_Clean_Signed.pdf

[8] B. Kerczewski, Spectrum for UAS control and non-payload communications, in Proc. IEEE Integrated Communs., Navigation and Surveillance Conf. (ICNS), 2013, pp. 121

[9] Medav, MEDAV RUSK Channel Sounder, http://www.channelsounder.de/.

[10] Rashdan, Ibrahim und Ponte Mueller, Fabian und Jost, Thomas und Sand, Stephan (2019), Measurement-based Geometrical Characterization of the Vehicle-to-Pedestrian Channel, EuCAP2019, 31,March-05. April. 2019, Krakow, Poland 